\title{
Functional osteoclast attachment requires inositol-1,4,5-trisphosphate receptor-associated cGMP-dependent kinase substrate
}

\author{
Beatrice B Yaroslavskiy, Irina Turkova, Yujuan Wang, Lisa J Robinson and Harry C Blair
}

Osteoclast activity is central to balanced bone turnover to maintain normal bone mass. A specialized osteoclast attachment to bone localizes acid secretion to remove bone mineral; in some cases, attachment is functionally impaired despite normal attachment proteins. The inositol-1,4,5-trisphosphate receptor-1 (IP3R1) is an intracellular calcium channel required for regulation of reversible osteoclast attachment by nitric oxide (NO), an important regulator of both normal and pathological bone degradation. In studies using human osteoclasts produced in vitro, we found that IP3R1 binds an endosomal isoform of the IP3R-associated cGMP-dependent kinase substrate (IRAG). IRAG is a substrate of cGMP-dependent kinase-1 (PKG1) and binds the PKG1 isoform PKG1 $\beta$, which was the predominant form of PKG1 in human osteoclasts. Western blots of IRAG were consistent with NO-dependent serine phosphorylation of IRAG.

An additional effect of PKG1 $\beta$ activity in osteoclasts was disassociation of IP3R1-IRAG complexes, as shown by analysis of IP3R1 complexes and by localization of the proteins within cells. IP3R1-IRAG complexes were stabilized by PKG or Src antagonists, Src activity being a requirement for IP3R1 calcium release downstream of PKG. IP3R1-mediated calcium release regulates cellular detachment in part through the calcium-dependent proteinase $\mu$-calpain. In osteoclasts with IRAG suppressed by siRNA, activity of $\mu$-calpain was increased relative to cells with normal IRAG, and regulation of $\mu$-calpain by NO was lost. Furthermore, cells deficient in IRAG detached easily from substrate and had smaller attached diameters and randomly distributed podosomes, although IRAG knockdown did not affect cell viability. Our results indicate that IRAG is required for PKG1 $\beta$-regulated cyclic calcium release during motility, and that disruption of the IP3R1-IRAG calcium regulation system is a novel cause of dysfunctional osteoclasts unrelated to defects in attachment proteins or acid secretion.

Laboratory Investigation (2010) 90, 1533-1542; doi:10.1038/labinvest.2010.120; published online 21 June 2010

KEYWORDS: endoplasmic reticulum; IP3R2; JAW1L; migfilin; osteoclast differentiation; VASP

Defects in osteoclasts cause osteopetrosis. Approximately half of these defects are because of abnormal acid secreting proteins, but many cases reflect abnormal cellular attachment, which in some cases occur despite normal attachment proteins. ${ }^{1}$ Osteoclast attachment and motility is regulated by nitric oxide (NO), mainly through stimulation of synthesis of cyclic GMP. In osteoclasts, the cyclic GMP-regulated protein kinase I (PKG1) modulates $\mathrm{Ca}^{2+}$ release by the inositol-1,4,5trisphosphate receptor-1 (IP3R1), an endoplasmic reticulum $\mathrm{Ca}^{2+}$ channel. This $\mathrm{Ca}^{2+}$ flux enables cell detachment for motility. $^{2}$ The IP3R1 is one of three homologous IP3Rs that mediate $\mathrm{Ca}^{2+}$ release gated by different stimuli; the IP3R2 also occurs in osteoclast precursors. ${ }^{3}$ It mediates $\mathrm{Ca}^{2+}$ oscillations important to osteoclast differentiation. ${ }^{4}$
In other cell types, IP3R1 co-precipitates with IP3R-associated cGMP-dependent kinase substrate (IRAG). IRAG is essential to cGMP regulation of $\mathrm{Ca}^{2+}$ release. ${ }^{5,6}$ The human gene encoding this protein is MRVI1, the homolog of a mouse gene, Mrvil, first identified at a murine retroviral integration site. The gene produces long or short proteins (Mrvila or Mrvilb), the long including an 85 amino acid $\mathrm{N}$-terminal extension that targets the gene product to the endoplasmic reticulum. ${ }^{7}$ Interaction of PKG1 with IRAG is limited to the PKG1 $\beta$ isoform of PKG1. It binds a motif present in both the long and short isoforms of IRAG. ${ }^{8}$ IRAG complexes with other proteins and, among other functions, regulates $\mathrm{PKG} 1 \beta$ nuclear translocation, ${ }^{9}$ but additional functions of IRAG are not well characterized.

Departments of Pathology and of Cell Biology and Physiology, University of Pittsburgh, and Veterans Affairs Medical Center, Pittsburgh, PA, USA

Correspondence: Professor HC Blair or Dr LJ Robinson, University of Pittsburgh, 705 Scaife Hall, Pittsburgh, PA 15261, USA.

E-mail: hcblair@imap.pitt.edu or robinsonlj@upmc.edu

Received 05 March 2010; revised 4 May 2010; accepted 4 May 2010 
Human IRAG protein (sometimes called Mrvil or JAW1L) is phosphorylated by PKGI $\beta$ near its C-terminus; ${ }^{10}$ this prevents $\mathrm{Ca}^{2+}$ transport initiated by inositol trisphosphate. ${ }^{11}$ Contrariwise, the open probability of human IP3R1 is increased by phosphorylation by Src-family kinases. ${ }^{12}$ Both PKG and Src are activated downstream of NO in osteoclast motility. ${ }^{2}$ IP3R1 is also a substrate for other kinases, ${ }^{13}$ which may be required for IP3R1 activation in some contexts. Our work $^{3}$ was consistent with counterregulatory effects of Src and PKG1 on IP3R1; tight regulation of $\mathrm{Ca}^{2+}$ release is important because short-term $\mathrm{Ca}^{2+}$ pulses mediate reversible events, but unopposed endosomal $\mathrm{Ca}^{2+}$ release causes apoptosis. ${ }^{14}$ However, the relationship of active PKG and its substrates in osteoclasts and how these affect shortterm $\mathrm{Ca}^{2+}$ channel activity were unclear.

In this study we performed protein localization and immune precipitation of IP3R1 and IRAG in osteoclasts under conditions in which PKG or $\mathrm{Ca}^{2+}$ activities were regulated. Our results suggest that IRAG is a negative regulator of IP3R1-mediated $\mathrm{Ca}^{2+}$ release, required for normal osteoclastic attachment, and that PKG activity causes IRAG to disassociate from IP3R1, whereas $\mathrm{Ca}^{2+}$ probably facilitates the association of IP3R1 and IRAG to restore basal conditions.

\section{MATERIALS AND METHODS Human Osteoclasts}

With institutional review board approval, human CD14 + cells were isolated as described ${ }^{15}$ by anti-CD14 immuno-magnetic selection after centrifugation on a density gradient to isolate cells with specific gravity $<1.077$. Osteoclast differentiation in vitro used recombinant human CSF1 and RANKL. ${ }^{16}$

\section{Reagents and PCR Protocols}

Three antibodies to IRAG were used, all from Santa Cruz Biotechnology (Santa Cruz, CA, USA); these were all polyclonal antibodies raised to human IRAG peptides: MRVI1 C-17 (sc-10958), a goat antibody to the C-terminal region, MRVI1a N-19 (sc-10953), a goat antibody recognizing the $\mathrm{N}$-terminal region found only on the long (endoplasmic reticulum) form of IRAG, and MRVI1a P-15 (sc-10954), an antibody to a central region of the molecule common to all known forms of the protein. Antibody to IP3R1 used for immune precipitation was also from Santa Cruz, and was raised to the C-terminal 20 amino acids of the mouse molecule (IP3R1 C-20, and sc-6093), or, for immune labeling or western blot, anti-IP3R1 rabbit polyclonal antibody to amino acids 1829-1848 of human IP3R1 (GTX25908; GeneTex, San Antonio, TX, USA). Antibody to IP3R phosphotyrosine353 was the kind gift of Andrew Marks (Columbia University, NY, USA) and was generated in rabbits using the phosphopeptide QEKMYpYSLVS. ${ }^{12}$ Oligonucleotide primers for quantitative PCR were: IP3R1 (from GenBank NM_001099952.1) forward $5^{\prime}$-TGCCTCAGTGAGAAAGAGCA- ${ }^{\prime}$ reverse $5^{\prime}$-GATCCCTG GGTTGAGAAACA-3' (209 base pairs); IP3R2 (GenBank
NM_002223.2) forward 5'-AGTCCAGTGCAGGATGGAAC-3' reverse $5^{\prime}$-TCTGCAGAAATGTATGGGCT- $3^{\prime}$ (233 base pairs). Human PKG1 (common region) forward $5^{\prime}$-TGAAGAACT TGGAGCTGTCGCAGA- $3^{\prime}$ reverse $5^{\prime}$-TCCTGGACCCATGG TACACAACTT-3' (179 base pairs). Human PKG1, variant 1 (PKG1 $\alpha$ ) (GenBank NM_001098512) forward 5'-AAACTC CACAAATGCCAGTCGGTG- $3^{\prime}$ reverse $5^{\prime}$-TCTGCGACAGCT CCAAGTTCTTCA-3' (220 base pairs). Human PKG1, variant 2 (PKG1 $\beta)$ (GenBank NM_006258) forward 5'-ACATCCA GGATCTCAGCCATGTGA-3' reverse $5^{\prime}$-ATCCACAATCTCC TGGATCTGCGA-3' (137 base pairs). Total RNA was isolated by oligo (dT) affinity. First-strand cDNA was synthesized from $1 \mu \mathrm{g}$ of RNA using random hexamer primers and Moloney murine leukemia virus reverse transcriptase. Real-time PCR used the SYBR green brilliant fluorescent DNA intercalating dye as analyte, purchased in a master mix containing nucleotides and buffer from Stratagene (La Jolla, CA, USA), adding $2.5 \mathrm{mM} \mathrm{Mg}, 100 \mathrm{nM}$ oligonucleotide primers, and first-strand cDNA. After $10 \mathrm{~min}$ at $95^{\circ} \mathrm{C}$, cycles of $15 \mathrm{~s}$ at $95^{\circ} \mathrm{C}$ and $1 \mathrm{~min}$ at $60^{\circ} \mathrm{C}$ were run an MX3000P thermocycler (Stratagene). The $\mathrm{Ca}^{2+}$ indicator fluo3 and the calpain substrate $t$-butoxycarbonyl-Leu-Met-chloromethylaminocoumarin (BOC) were from Molecular Probes (Carlsbad, CA, USA). The Src inhibitor 4-amino-5-(4-chlorophenyl)-7-(t-butyl) pyrazolo[3,4-d]pyrimidine (PP2) and its inactive congener 4-amino-7-phenylpyrazol[3,4-d] pyrimidine (PP3) were from Calbiochem (San Diego, CA, USA). The NO donor sodium nitroprusside (SNP) was from Sigma (St Louis, MO, USA). The hydrolysis-resistant cGMP activator 8-(4-chlorophenylthio)guanosine- $3^{\prime}, 5^{\prime}$-cyclic monophosphate (8-pCPT-cGMP), and the inactive blocking cGMP analogs Rp-8-Br-guanosine- $3^{\prime}, 5^{\prime}$-cyclic phosphorothioate (Rp-8-BrcGMPS), 8-(Rp-4-chlorophenylthio)guanosine-3' $5^{\prime}$-cyclic phosphorothioate (Rp-CPT-cGMPS), and $\beta$-phenyl-1-N2-etheno-8bromoguanosine- $3^{\prime}, 5^{\prime}$-cyclic phosphorothioate (Rp-8BrPET-cGMPS) were from Biolog (Bremen, Germany). The $\mathrm{Ca}^{2+}$ chelator 1,2-bis(2-aminophenoxy)ethane-N,N,N',N'tetraacetate, acetoxymethyl ester, was from Molecular ProbesInvitrogen (Carlsbad, CA, USA). Monoclonal anti-phosphotyrosine was from Cell Signaling (Beverly, MA, USA). Polyclonal anti-phosphoserine was from Abcam (Cambridge, MA, USA) and was raised in rabbits using a mixture of phosphoserine peptides (ab9332). Polyclonal anti-Src was from Santa Cruz. Polyclonal anti-PKGI was from Stressgen (Victoria, BC, Canada). Anti- $\beta$ actin was from Sigma.

\section{Flow Cytometry}

Flow cytometry was performed as described ${ }^{17}$ with minor modifications. In brief, after washing in phosphate-buffered saline with $0.1 \%$ bovine serum albumin and $0.1 \% \mathrm{NaN}_{3}$, aliquots of $3 \times 10^{5}$ cells were incubated for $30 \mathrm{~min}$ on ice with Fluor-conjugated mouse monoclonal antibodies (FITC-labeled anti-CD3, PE-labeled anti-CD14, and isotype controls (all from BD Biosciences PharMingen, San Diego, CA, USA) and Cy-5-labeled anti-CD19 (Invitrogen, Carlsbad, 
CA, USA)) diluted in PBA. After three washes and fixation in $2 \%$ paraformaldehyde, cells were evaluated on a FACScalibur instrument using Cell Quest Software (BD Biosciences PharMingen) for data analysis.

\section{Western Analysis and Immunoprecipitation}

For western blots, cells were lysed in $0.5 \%$ octyl phenoxylpolyethoxyethanol (NP-40), 1\% polyoxyetheylene octyl phenyl ether (Triton X-100), $150 \mathrm{mM} \mathrm{NaCl}, 20 \mathrm{mM}$ tris, $\mathrm{pH}$ 7.5 , with proteinase inhibitors in phosphorylation studies, with phosphatase inhibitors $(100 \mu \mathrm{M} \mathrm{NaF}, 1 \mathrm{mM}$ sodium orthovanadate). Proteins were separated on SDS-PAGE and transferred to polyvinylidine membranes for immune labeling with peroxidase-coupled secondary antibodies and enhanced chemiluminescence detection (ECL plus; Thermo Scientific, Waltham MA, USA). ${ }^{15}$ Unless otherwise specified, separations used 9\% SDS-PAGE gels. Where blots were reprobed, they were stripped of previous antibodies using Restore (Pierce, Rockford, IL, USA). For immunoprecipitation, cells were lysed in $0.1 \%$ NP-40 detergent with phosphatase and protease inhibitors, and lysates were centrifuged to remove debris and pre-cleared with protein $A / G$ plus (Santa Cruz) and then incubated overnight at $4{ }^{\circ} \mathrm{C}$ with antibody. Antibody-bound proteins were recovered using protein A/G beads (Miltenyi Biotech, Auburn, CA, USA) by centrifugation after $2 \mathrm{~h}$ of incubation. The precipitated beads were washed five times with NP-40 lysis buffer and eluted in Laemmli buffer for western blot analysis.

\section{In Situ Immune Labeling}

After indicated treatment of cells, cell cultures were fixed in $2 \%$ formaldehyde in phosphate-buffered saline for $10 \mathrm{~min}$, and then kept in ethanol at $-20{ }^{\circ} \mathrm{C}$ until used. Labeling was performed at room temperature after $10 \mathrm{~min}$ in $0.2 \%$ polyoxyethylene octyl phenyl ether (Triton X-100, a nonionic detergent; Sigma-Aldrich, St Louis, MO, USA) in phosphatebuffered saline for permeabilization. Cultures were then incubated for $10 \mathrm{~min}$ in blocking buffer (1\% bovine serum albumin with $5 \%$ goat serum in phosphate-buffered saline and $0.05 \%$ polyoxyethylene sorbitol (Tween 20 , a surfactant). Cells were then incubated for $1 \mathrm{~h}$ with the primary antibodies, described above, in blocking buffer. Cells were washed and then incubated for $1 \mathrm{~h}$ with secondary antibodies in phosphate-buffered saline: these were Cy3-labeled donkey anti-goat IgG, at 1:500, AlexaFluor488-labeled donkey anti-mouse IgG, at 1:250 (from Invitrogen), and Cy3- or FITC-labeled donkey anti-rabbit IgG, at 1:500 (Jackson ImmunoResearch, Westgrove PA, USA). Controls omitting primary antibody were performed to identify nonspecific staining. For nuclear labeling, Hoechst 33342 blue (Invitrogen) was used at $10 \mathrm{ng} / \mathrm{ml}$ in $140 \mathrm{mM} \mathrm{NaCl}$.

\section{RNA Interference}

Cells were transfected with siRNA targeting two PKGI sequences, or two IRAG sequences as described. ${ }^{15}$ Cells were transfected using siPORT Amine transfection reagent (Ambion, Austin, TX, USA), a blend of polyamines. Controls used transfection with nonsense siRNA. Sequences were screened for homology to other proteins using BLAST (www.ncbi.nlm.nih.gov/BLAST). The siRNAs for this work targeted PKGI sequences from Genbank Z92867, + 109-129 from the start codon, $5^{\prime}$-AAGAGGAAACTCCACAAATGC- $3^{\prime}$ and 124-46, 5'-AAATGCCAGCGGTGCTCCCAGT-3'. RNA duplexes were synthesized at Integrated DNA Technologies (Coralville, IA, USA) targeting these sequences. Transfection used mixtures of siRNAs with $100 \mathrm{nM}$ total siRNA. For IRAG silencing, siRNAs to the large transcript of IRAG were purchased from Santa Cruz as a mixture of $100 \mathrm{nM}$ total siRNA targeting two sequences $5^{\prime}$-UGGAUUUGACUUGUCCUU UTT- $3^{\prime}$ and $5^{\prime}$-AAAGGACAAGUCAAAUCCATT- $3^{\prime}$ of the long isoform of human IRAG (MRVI1). To visualize transfection, Cy3 was covalently attached to the duplex siRNA (Silencer siRNA labeling kit; Ambion).

\section{Digital Imaging}

Images were acquired using a Nikon TE3000 phase-fluorescence microscope with a 14 bit $2048 \times 2048$ element CCD (Diagnostic Instruments, Sterling Heights, MI, USA). Phase or transmitted light microscopy used a NA $0.70 \times 40$ objective with red, green, and blue filters to assemble color images. Fluorescence images used 1.4 NA $\times 40$ or $\times 100$ oil objectives. Blue fluorescence used excitation at $380-400 \mathrm{~nm}$, a $430 \mathrm{~nm}$ dichroic mirror, and $430-480 \mathrm{~nm}$ emission filter; green used $450-490 \mathrm{~nm}$ excitation, a $510 \mathrm{~nm}$ dichroic mirror, and a $500-570 \mathrm{~nm}$ emission filter; and red used $530-560 \mathrm{~nm}$ excitation, a $575 \mathrm{~nm}$ dichroic mirror, and $580-650 \mathrm{~nm}$ emission filter. Intracellular $\mathrm{Ca}^{2+}$ was studied using fluo3. Cells were incubated for $20 \mathrm{~min}$ at $37^{\circ} \mathrm{C}$ in $10 \mathrm{mM}$ of membrane-permeant fluo-3 acetoxymethyl ester (AM) and, after washing cultures, epifluorescence images were acquired using excitation $450-490 \mathrm{~nm}, 510 \mathrm{~nm}$ dichroic mirror, and $520 \mathrm{~nm}$ barrier filter. For measurement of $\mathrm{Ca}^{2+}$-dependent proteinase (calpain) activity, $50 \mu \mathrm{M}$ of the coumarin-conjugated substrate BOC was added for 20 min to osteoclasts on glass coverslip culture dishes. Fluorescence intensity was determined by imaging of the activated substrate in cells using the green channel. For colocalization of IRAG and IP3R1, we performed digital coincidence analysis using red and green images with a hue-saturation-intensity filter (Fovea Pro, Reindeergraphics, Asheville, NC, USA) in images adjusted to equal red-green saturation (Figure 3, color images), selecting pixels containing red and green $\left(0-45^{\circ}\right)$, at any saturation, with intensity above background (in most cases $>40 / 256$ bits; Figure 3, monochrome images).

\section{RESULTS}

\section{IP3R1 and IP3R2 in Human Osteoclasts}

IP3Rs regulate differentiation, death, and activity in cells including lymphocytes and monocytes. ${ }^{18}$ Kuroda et $a l^{4}$ reported that peripheral blood mononuclear cells lacking 
IP3R1 differentiated into osteoclasts whereas cells lacking IP3R2 failed to differentiate normally, although we reported that motility in osteoclasts depends on $\mathrm{Ca}^{2+}$ regulated by IP3R $1 .{ }^{2}$ We studied the expression of IP3R1 and IP3R2 in CD14 cells and osteoclasts (Figure 1). Cells before and after CD14 selection were evaluated by flow cytometry (Figure 1a). After CD14 selection, T or B cells, labeled by CD3 or CD19, represented $<2 \%$ of total cells (Figure 1a). From this population of mononuclear cells, essentially all differentiated to express tartrate-resistant acid phosphatase (TRAP) after 2 weeks in CSF-1 and RANKL (Figures $1 \mathrm{~b}$ and $\mathrm{c}$ ). The expression of IP3R1 and IP3R2 mRNAs in CD14-selected cells and in osteoclasts from these cells were determined by quantitative PCR. In monocytic precursors mRNAs for IP3R1 and IP3R2 were present in similar quantities.

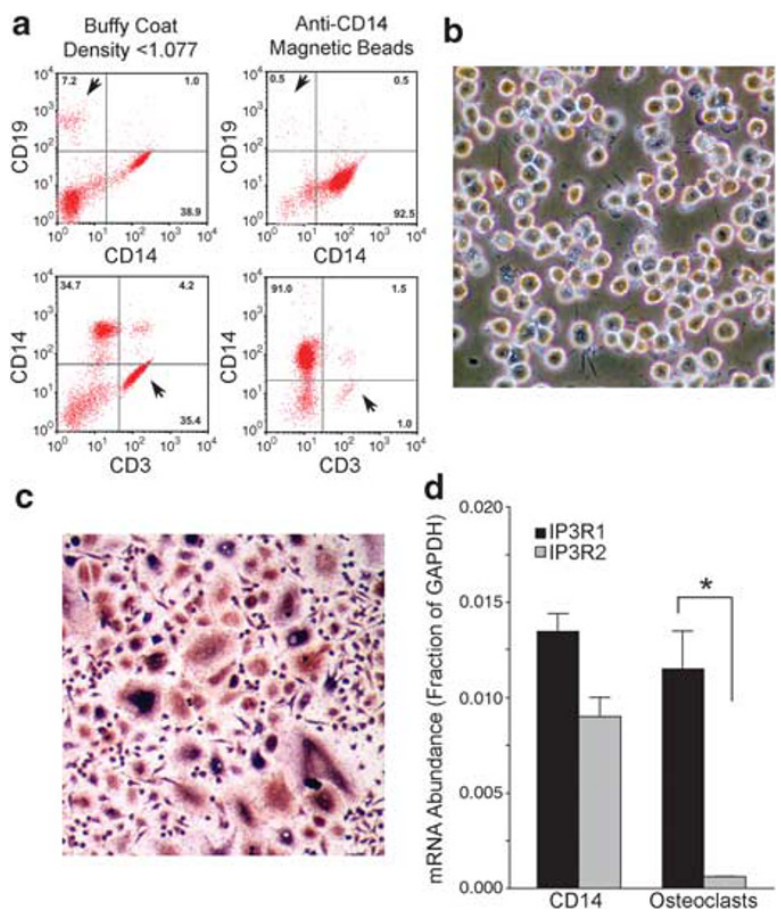

Figure 1 Isolation of CD14 osteoclast precursors, differentiation of osteoclasts in vitro, and expression of IP3R1 and 2 in CD14 cells and osteoclasts. (a) Peripheral blood mononuclear cells (left) and CD14-selected cells (right) were evaluated for CD14, CD3, and CD19 expression by flow cytometry. Affinity-purified CD14 cells (right column) contained 1 to $2 \%$ CD19 or CD3 lymphocytes, with marked enrichment for monocytes. The following mouse monoclonal antibodies were used: FITC-labeled antihuman CD14 from BD Biosciences PharMingen, Cy-5- labeled CD19 from Invitrogen, and PE-labeled CD3 from Caltag (Burlingame, CA, USA).

(b) CD14-purified cells appeared by phase microscopy as monotonous mononuclear cells. The field is $180 \mu \mathrm{m}^{2}$. (c) Osteoclasts, stained for TRAP activity, from CD14 cells after 2 weeks in $40 \mathrm{ng} / \mathrm{ml}$ RANKL and $10 \mathrm{ng} / \mathrm{ml}$ CSF-1. Nearly all show TRAP positivity and the majority of the cell nuclei are fused into giant cells. Transmitted light photograph, the field is $350 \mu \mathrm{m}^{2}$. (d) Expression of IP3R1 and IP3R2 in CD14-selected cells and osteoclasts by quantitative PCR. Messenger RNAs for both IP3R1 and IP3R2 were present. IP3R1 was the predominant transcript in differentiated cells, approximately tenfold greater signal than IP3R2 $\left({ }^{*} P<0.05\right)$.
In osteoclasts, IP3R1 was expressed at approximately tenfold the level of IP3R2 (Figure 1d). Each of the IP3Rs is regulated by complex inositol and kinase-dependent pathways; it is not surprising that multiple forms are expressed during differentiation, with different functions for each isoform. However, the IP3R1 has been identified in complexes with the PKG1 $\beta$-binding regulatory protein IRAG ${ }^{8}$ and hence the involvement of IP3R1 in PKG-dependent regulation is not surprising. We detected IP3R1 but not IP3R2 in osteoclasts by unamplified screening, ${ }^{2}$ probably because of the relatively small amount of IP3R2 mRNA.

\section{IRAG and PKG Isoforms in Osteoclasts}

Consistent with analysis of IRAG homologs in mice and humans, ${ }^{7}$ two forms of IRAG with apparent sizes of $\sim 100$ and $150 \mathrm{kDa}$ were present. The relative amounts of the two isoforms was unaffected by NO or cGMP activating or inhibiting treatments, by western blot (Figure 2a). IRAG is a target for PKG1 $\beta$ phosphorylation at ser664 and ser667. ${ }^{10}$ Anti-phosphoserine labeling showed that the large form of IRAG had increased phosphoserine after $10 \mathrm{~min}$ of exposure to the NO donor SNP, but little phosphoserine after exposure to a cGMP antagonist (Figure 2b). Serine phosphorylation of the small form of IRAG was not studied; only the large form of IRAG is associated with IP3R1 (see below). Because IRAG contains a recognition sequence specific for the PKG isoform $\operatorname{PKG} 1 \beta$, we determined the expression of $\operatorname{PKG} 1 \alpha, \operatorname{PKG} 1 \beta$, and PKG1 (total) in osteoclasts (Figure 2c). The quantity of PKG1 $\beta$ mRNA relative to GAPDH was indistinguishable from that of PKG1 (total); PKG1 $\alpha$ mRNA was present, but was only $\sim 5 \%$ of the total PKG1 mRNA, and significantly less than either the total PKG1 or PKG1 $\beta$ isoform $(P<0.05)$. The reason for specificity for the $\beta$ isoform of the transcript in these cells is not known; a small part of the difference in Figure $2 \mathrm{c}$ might reflect different efficiency of amplification of the PKG1 $\alpha$ and $1 \beta$ probes, but the slopes of the amplification curves were essentially identical (not shown), indicating that probe-specific differences in PCR efficiency were probably insignificant.

\section{IP3R1-IRAG Localization in Osteoclasts}

We examined IP3R1 and IRAG by in situ labeling of human osteoclasts differentiated on glass coverslips (Figure 3), with PKG inhibited (top panels) or activated (bottom panels). As IP3R1 increases cytoplasmic $\mathrm{Ca}^{2+}$, causing secondary effects, the experiment was performed without (Figure 3a) or with (Figure 3b) the cell-permeant $\mathrm{Ca}^{2+}$ chelator 1,2-bis (2-aminophenoxy)ethane-N,N,N',N'-tetraacetate (BAPTA) added $40 \mathrm{~min}$ before the PKG-modifying agents. Pixels labeled both for IRAG and IP3R1 were determined by digital coincidence (monochrome panels, Figures $3 \mathrm{a}$ and $\mathrm{b}$, right panels). Whether $\mathrm{Ca}^{2+}$ was allowed to vary, there was colocalization of IP3R1 in an endosomal-perinuclear pattern when PKG was inhibited. Colocalization of IRAG and IP3R1 was inhibited by PKG activation. The difference was larger in 
Precipitate: IRAG (internal) Blot IRAG (internal)

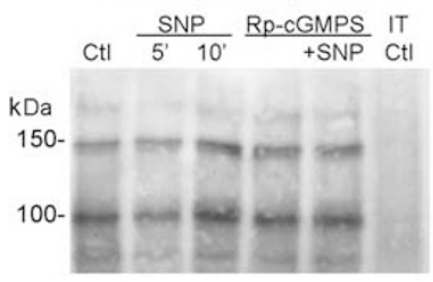

b

Precipitate: IRAG (internal)

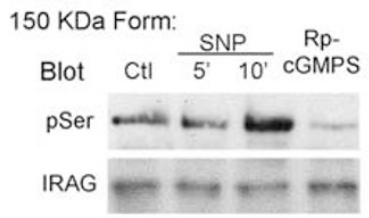

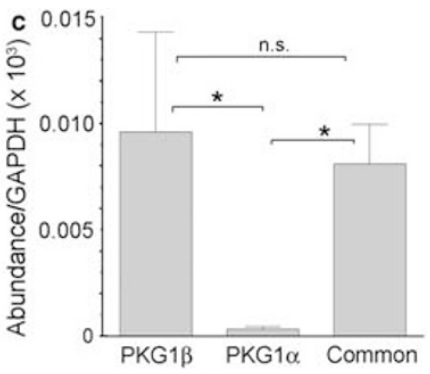

Figure 2 The IP3R-associated CGMP-dependent kinase substrate (IRAG) and the IRAG-binding isoform of PKG1 in osteoclasts. (a) Western blotting for IRAG was performed on anti-IRAG immunoprecipitates from osteoclasts that were untreated $(\mathrm{CtI})$ or treated with the NO donor sodium nitroprusside (SNP) at $100 \mu \mathrm{M}$ for 5-10 min, or with the inhibitory cGMP analog 8-(Rp-4-chlorophenylthio)guanosine-3', $5^{\prime}$-cyclic phosphorothioate (Rp-cGMPS), $50 \mu \mathrm{M}$, for 30 min with or without subsequent treatment with SNP (100 $\mu \mathrm{M}$ for $10 \mathrm{~min})$. Western blotting showed similar amounts of the large and small forms of IRAG, with $\mathrm{Mr}$ of $\sim 150$ and $100 \mathrm{kDa}$, under all conditions. The last lane shows an isotype control (nonimmune serum). (b) Anti-phosphoserine blot (upper panel) of anti-IRAG immunoprecipitates from untreated osteoclasts (Ctl) or osteoclasts treated with $100 \mu \mathrm{M}$ SNP for 5-10 min, or with $50 \mu \mathrm{M}$ Rp-cGMPS for $30 \mathrm{~min}$. The blots were then stripped and reprobed with anti-IRAG antibody to confirm similar recovery of IRAG (lower panel). IRAG phosphoserine was increased after treatment with SNP, but reduced by the PKG antagonist. (c) Relative expression of PKG1 isoforms in osteoclasts. Quantitative PCR using primers specific to PKG1 $\beta$ or PKG1 $\alpha$ or recognizing both forms (Common) was performed. The amount of product for each primer set is shown relative to GAPDH as an internal control. The amount of PKG1 $\beta$ was indistinguishable from total PKG1. PKG1 $\alpha$ mRNA was present, but at levels significantly lower than PKG1 $\beta\left({ }^{*} P<0.05\right.$; NS, not significant).

a
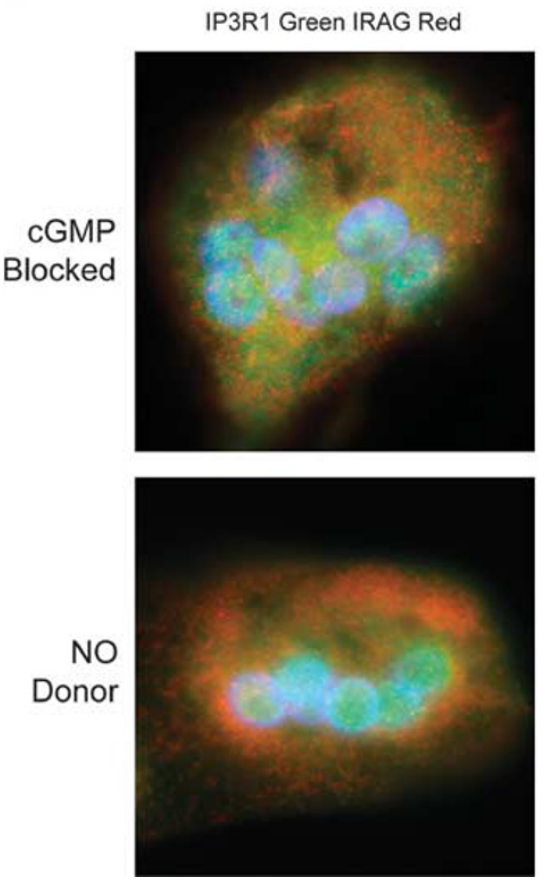

Coincidence
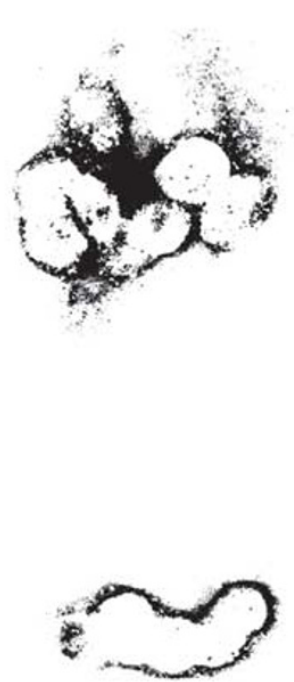

b
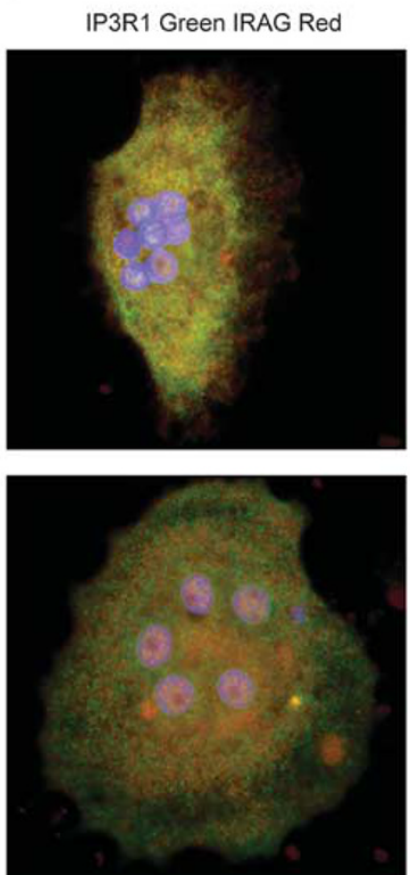

Coincidence
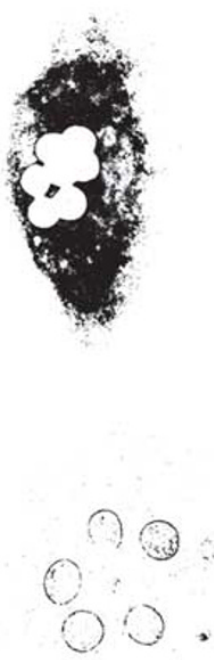

Figure 3 Localization of IRAG at endosomal sites. Photomicrographs of human osteoclasts on glass coverslips with immune labeling as indicated. Antibody N19 (see Materials and methods) recognizing the $150 \mathrm{kDa}$ form of IRAG was used. To determine colocalization of IP3R1 without subjectivity, labeling of pixels for both IRAG and IP3R1 were calculated by digital coincidence selection (see Materials and methods); the results are shown in black in columns labeled Coincidence. Colocalization was more prominent when PKG was inhibited by Rp-cGMPS, than when PKG was activated by NO, and this difference was accentuated when $\mathrm{Ca}^{2+}$ was low. All fields shown are $50 \mu \mathrm{m}^{2}$. (a) IP3R1 and IRAG in human osteoclasts on glass coverslips with PKG inhibited by $50 \mu \mathrm{M}$ Rp-cGMPS (30 min of incubation; top row of panels) or activated by $100 \mu \mathrm{M}$ sodium nitroprusside (10 min of incubation, bottom row of panels). (b) The same experiment was performed after pre-treatment with the cell-permeant $\mathrm{Ca}^{2+}$ chelator-donor, 1,2-bis(2-aminophenoxy)ethane- $\mathrm{N}, \mathrm{N}, \mathrm{N}^{\prime}, \mathrm{N}^{\prime}$ tetraacetate (BAPTA, $70 \mu \mathrm{M}$, as acetoxymethyl ester) added 40 min before the PKG effectors.

$\mathrm{Ca}^{2+}$-chelated cells (Figure 3b), suggesting that IRAG-IP3R1 association is sensitive to the $\mathrm{Ca}^{2+}$ signal activated by NO. Because of the clearer difference when $\mathrm{Ca}^{2+}$ was held at low levels, further work on IP3R1 and IRAG complexes was carried out using BAPTA pre-treated cells unless specified. 


\section{PKG and IRAG Localization at Other Cell Sites}

As we reported, ${ }^{16}$ PKG did not localize clearly with any cellular structure, with IRAG or with IP3R1 (not illustrated). This may reflect that the dwell time for PKG, including at its phosphorylation sites, is too short to permit localization. In contrast, surveys of IRAG labeling also showed localization at additional cell structures. Antibodies reacting with both short and long forms of IRAG, after NO donor activation, labeled IRAG at cellular attachments, visualized with phalloidin (Figure 4a). The effect was not observed when repeated with antibodies specific for the large (endosomal) type of IRAG (not illustrated). In an earlier work, we found the PKG target protein VASP at osteoclast membrane attachments, which was associated with the organizing protein migfilin when PKG was activated. ${ }^{15}$ To determine whether the cell surface localization of IRAG might reflect membrane-associated protein complexes, we examined immune precipitates of IP3R1, and precipitates of IRAG from supernatants after IP3R1 immune precipitation, for migfilin and VASP (Figure 4a). IRAG that was not precipitated with IP3R1 was associated with migfilin and VASP. This association was increased by SNP. The association of IRAG with these membraneregulating proteins after precipitation of IRAG bound to IP3R1 suggests a role for the non-endosomal type of the IRAG in the regulation of cell attachment. In NO donor- treated cells, there was, in addition to reduced endoplasmic reticulum IRAG, strong nuclear localization of IRAG. This is visible in Figure 3, but it is observed clearly with labeling limited to nuclei and IRAG (Figure 4b). This nuclear localization required PKG1, as shown by siRNA knockdown, which eliminated nuclear redistribution of IRAG in SNPtreated cells (Figure 4c).

\section{Inhibition of IRAG Expression Increases Intracellular $\mathrm{Ca}^{2+}$ and Decreases Cell Spreading}

We used siRNAs to reduce expression of the large form of IRAG (Figure 5a); this reduced the large IRAG 70\%; the siRNAs do not bind the alternate short mRNA for IRAG. The siRNA suppression caused an increase in average $\mu$-calpain activity in unstimulated cells assayed using BOC (Figure 5b, bars 1 versus 4 ). In cells transfected with control siRNA, NO stimulated calpain activity whereas PKG inhibition reduced it compared with unstimulated cells, as expected. But in IRAGdeficient cells with high basal calpain activity, NO donors had no additive effect. However, calpain activation still required PKG, as incubation in Rp-cGMPS for 45 min before BOC addition resulted in similar low activity in cells with normal IRAG or in cells with IRAG suppressed. In keeping with the effect of IRAG inhibition on calpain activity, $\mathrm{Ca}^{2+}$, by Fluo-3 fluorescence, was higher in IRAG-suppressed than a

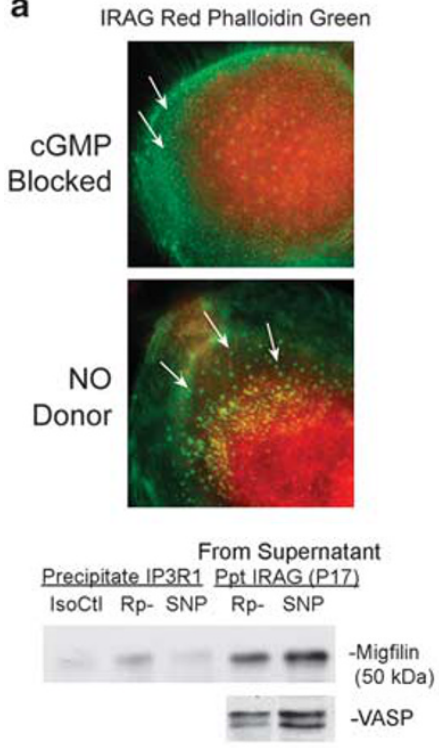

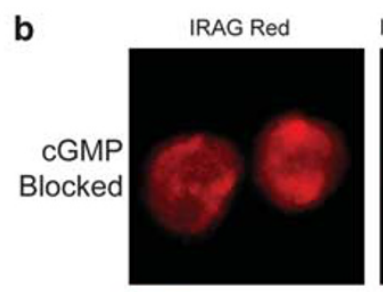
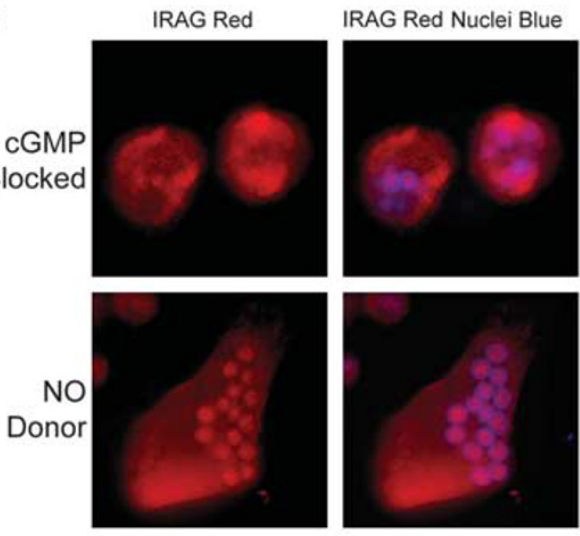

c
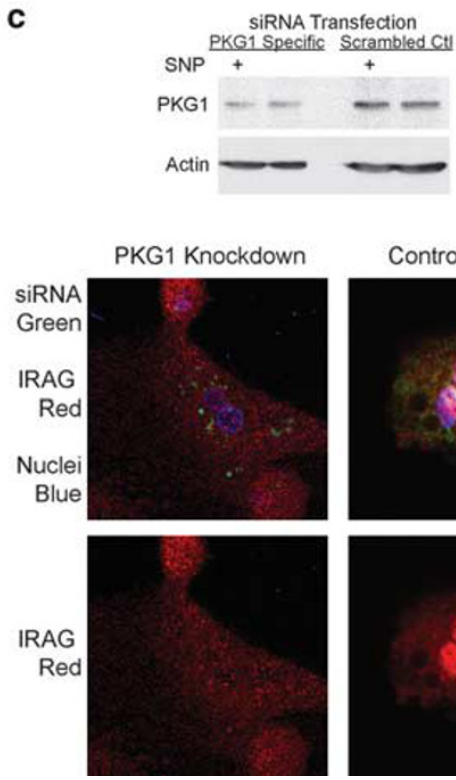

Control siRNA

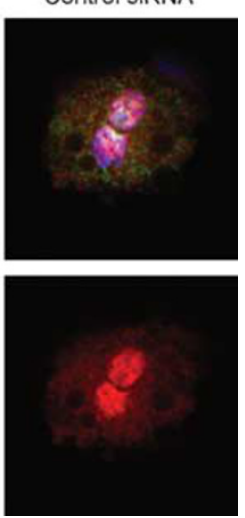

Figure 4 Effects of NO donors on distribution of IRAG at non-endosomal sites. (a) At the plane of cell attachment to glass substrate, antibody reacting with long and short forms of IRAG (red) did not localize with punctate cellular attachments (top, phalloidin, green; arrows). However, after NO donor activation, IRAG localized in cellular attachments (bottom, yellow; arrows). Fields are $30 \mu \mathrm{m}^{2}$. IP3R1 precipitates from osteoclasts treated or not with NO donor or PKG inhibitor and IRAG precipitates from supernatants after IP3R1 precipitation were blotted for migfilin and VASP (bottom panels). In IP3R1 precipitates (lanes 1-3) there was no significant migfilin. After depletion of IP3R1, precipitates of supernatant IRAG included migfilin. This association was increased by sodium nitroprusside. This fraction also included VASP, which also increased with sodium nitroprusside. VASP in IP3R1 precipitates was not determined. (b) In cells treated with NO donors, IRAG nuclear localization was increased relative to cells in which PKG was blocked. Similar effects were observed using activating cGMP analogs (not illustrated). Fields are $50 \mu \mathrm{m}^{2}$. (c) Effect of PKG knockdown on redistribution of IRAG to nuclei. PKG siRNA reduced PKG expression by approximately $80 \%$ (top); the amount of PKG was not affected by sodium nitroprusside (100 $\mu \mathrm{M}$ treatment, 30 min before cell lysis). In PKG-silenced cells (siRNA shown by green label), after sodium nitroprusside treatment (100 $\mu \mathrm{M}, 10$ min), IRAG was no longer redistributed to nuclei (bottom left), whereas in cells with control siRNA, also after sodium nitroprusside treatment, nuclear localization was strong (bottom right). 
a

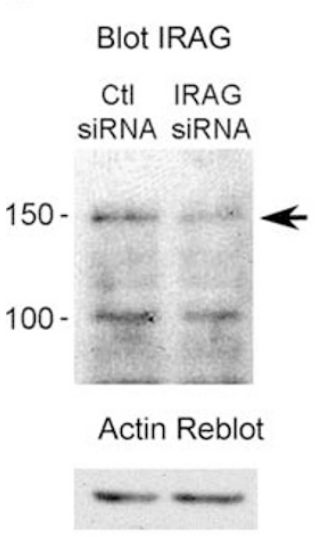

b (1) Nonsense siRNA

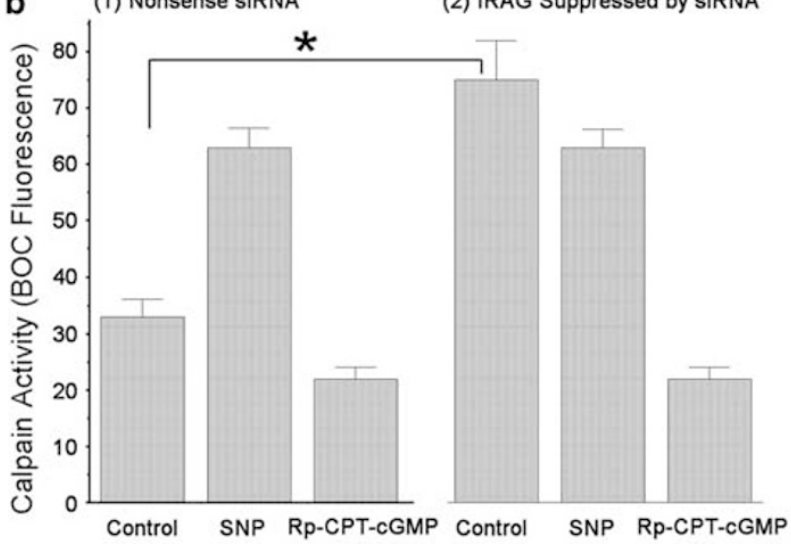

C

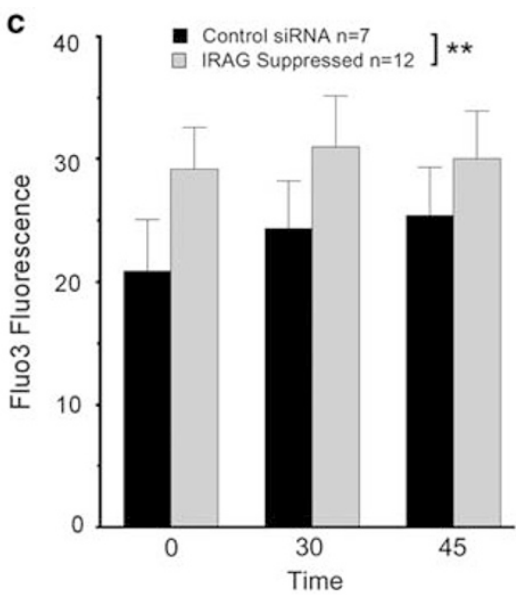

Figure 5 Inhibition of IRAG expression by siRNA and effects on calpain activity and relative intracellular $\mathrm{Ca}^{2+}$. (a) Suppression of expression of the large form of IRAG by $72 \mathrm{~h}$ of incubation after transfection of a mixture of two siRNAs; this reduced the amount of the $150 \mathrm{kDa}$ form of IRAG to $\sim 30 \%$ of control by western blot for total IRAG protein. Changes in the small form of IRAG were not observed, which is expected as the siRNAs targeted the long transcript and the target sequences are not present in the short isoform. This was intentional in that the long form only localizes to the endosomal membranes (see text). (b) When IRAG is suppressed, basal $\mathrm{Ca}^{2+} /$ calpain is increased, but it is no longer sensitive to NO. Suppression of IRAG increased average cellular $\mu$-calpain activity in the absence of stimulation of PKG (bar 1 versus $4,{ }^{*} P<0.05$ ). Preparations incubated 45 min with the strong PKG antagonist Rp-CPTcGMPS, $50 \mu \mathrm{M}$, showed low calpain activity in both control and IRAG-suppressed cells. When sodium nitroprusside was added to IRAG-suppressed or control cells, differences in calpain activity were not significant (bar 2 versus 5). Calpain activity was assayed using $t$-butoxycarbonyl-Leu-Metchloromethylaminocoumarin (BOC), which when cleaved by calpain generates a fluorescent signal. ${ }^{2}$ (c) Suppressing IRAG increased average Ca ${ }^{2+}$ in osteoclasts. Calcium was determined using Fluo-3, measuring fluorescence at $520 \mathrm{nM}$. Fluorescent signal were measured relative to no-cell background at 0 , 30, and $45 \mathrm{~min}$, all of which gave similar results. Differences were consistent, with $P<0.01$ by analysis of variance for control versus IRAG-suppressed cells. Mean \pm s.e.m., $n=7$ (control, black) and $n=12$ (IRAG-suppressed cells, gray).

in control cells (Figure 5c). There was considerable cell-tocell variability, but the differences between groups were consistent and significant in consecutive assays at 0,30 , and $45 \mathrm{~min}$. Labeling of individual cells for siRNA transfection (Figure $6 \mathrm{a}$ ) showed that $\sim 70 \%$ of individual cells were transfected, in keeping with the reduction of IRAG (Figure 5a). Cells with inhibited IRAG production had reduced cell diameters (Figure 6b), although no change in cell density or other evidence of cell death was observed (Figure $6 c)$. The effect on cell diameter was consistent over several experiments, and the effect was increased by SNP (not illustrated). This is a qualitative, and important, change in the ability of the cells to regulate their attachment. Furthermore, we labeled the podosomes of control and IRAG-deficient cells (Figure 6d) that showed a random distribution of podosomes in the knockdown cells, whereas control cells had the rings of podosomes characteristic of osteoclast attachment. ${ }^{1}$

\section{The Effect of PKG Activators and Inhibitors on IP3R1-IRAG Association}

Immune precipitation of IP3R1 was performed, followed by western analysis for IRAG, using untreated cells or cells pretreated with SNP or cGMP analogs. The $\sim 150 \mathrm{kDa}$ form of IRAG precipitated with IP3R, which was not surprising in that this form of IRAG encodes an N-terminal that includes an endoplasmic reticulum recognition site (Figure 7a). This was consistent with colocalization of the proteins in cells (Figure 3). Interestingly, and also in keeping with the immune localization, the IRAG-IP3R1 association was inhibited by the NO donor SNP. However, the Src antagonist PP2, but not its inactive congener PP3, stabilized the complex, even with SNP added. Control blots confirmed similar quantities of IRAG in lysates of these cell preparations (Figure 7a). Dependency on PKG activation was confirmed by comparing the effects of activating and inhibiting hydrolysis-resistant cGMP analogs, with cell $\mathrm{Ca}^{2+}$ held at a low level using BAPTA (Figure 7b). Furthermore, PKG-dependent tyrosine kinase phosphorylation of IP3R1 was studied using antibody to IP3R1 phosphotyrosine353. This showed tyrosine phosphorylation ${ }^{12}$ under conditions activating $\mathrm{Ca}^{2+}$ release (Figure 7c). This was consistent with results showing that elimination of PKG1 prevents Src phosphorylation after 8 -Br-cGMP treatment. ${ }^{2}$

\section{DISCUSSION}

Osteoclasts express several $\mathrm{Ca}^{2+}$ channels, including IP3Rs, ryanodine receptors, and the cell membrane $\mathrm{Ca}^{2+}$-activated $\mathrm{Ca}^{2+}$ channel Orai. ${ }^{19}$ The functions of the ryanodine receptor and Orai in osteoclasts are not well characterized. IP3R2 is active in osteoclast differentiation under some conditions, ${ }^{4}$ whereas IP3R1 is differently regulated and, specifically, is required for NO-regulated motility. ${ }^{2}$ Earlier work suggested a single major type of PKG1 in osteoclasts. ${ }^{20}$ In this study we found that IP3R1 is the major IP3R, and that PKG1 $\beta$ is the predominant isoform of PKG1, in mature human osteoclasts. PKG1 $\beta$ regulates IP3R1, in part, through the large, endosomal type of IRAG, which incorporates a 
a siRnA Red, Phase Green

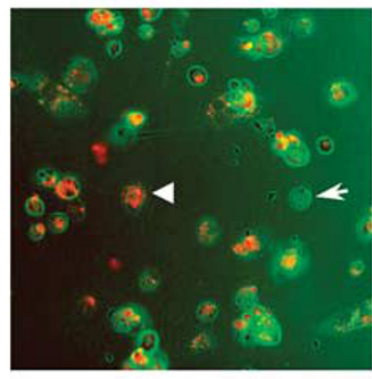

C (1) SIRNA IRAG

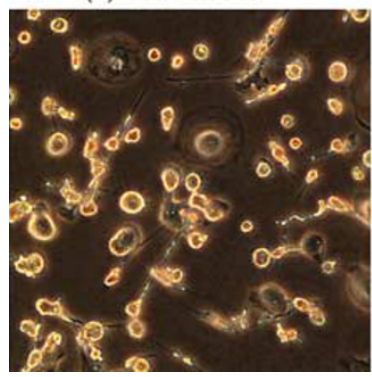

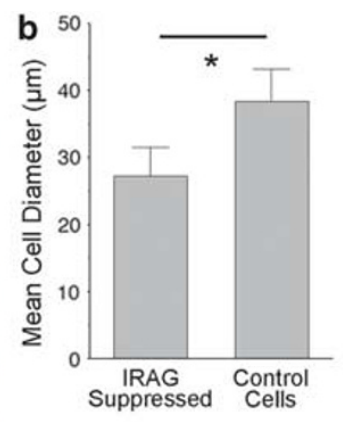

(2) Control siRNA

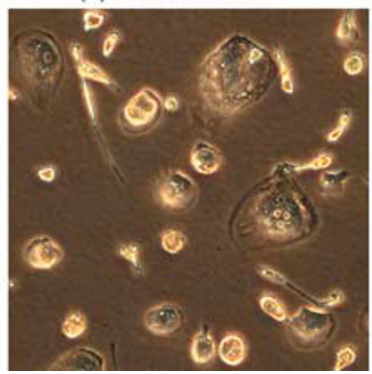

d
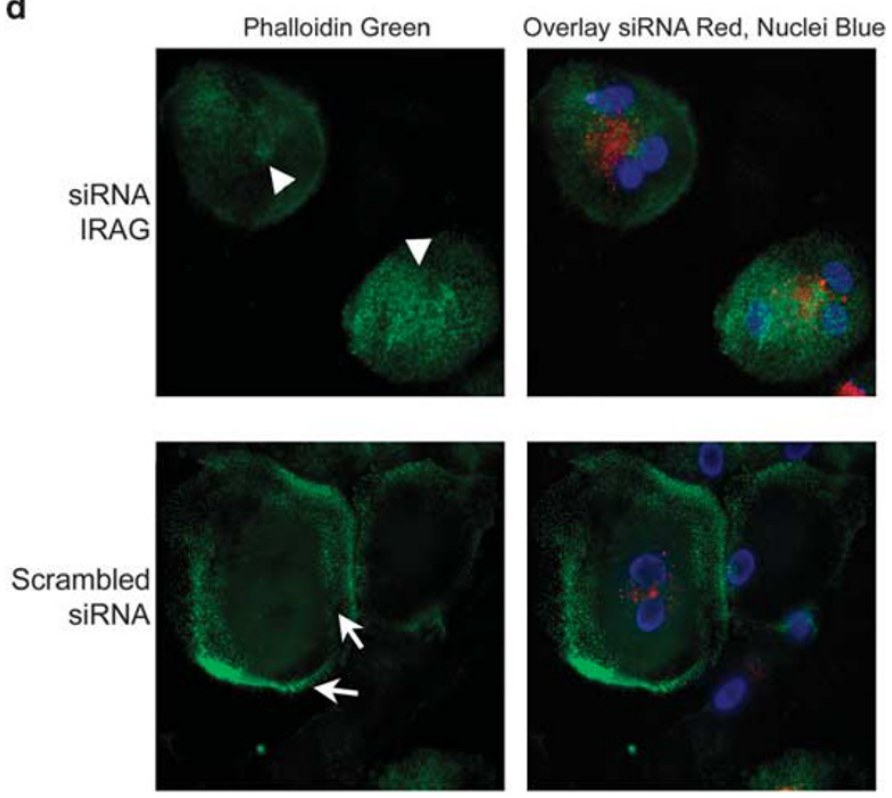

Figure 6 Transfection of siRNA to reduce IRAG expression reduced cell spreading. (a) Phase photograph of osteoclasts (green) after transfection with fluorescently tagged siRNA (red). Approximately $70 \%$ of cells are labeled, indicating the transfection efficiency. (b) Cells with inhibited IRAG production had mean cell diameter reduced by $\sim 30 \%$; the difference is significant $(P<0.05) ; n=10$. (c) Phase photomicrographs of osteoclast cultures after transfection with IRAG-specific siRNA (1, left) or control siRNA (2, right). No increase in floating or apparently apoptotic cells were observed in IRAG suppression, although cell spreading was reduced. Photographs in (a) and (c) are of $220 \mu \mathrm{m}^{2}$ fields. (d) Photomicrographs of control and IRAG-specific siRNA-transfected cells, as in (a) (red), with additional labeling using phalloidin (green) to show podosomes and Hoechst (blue) to label nuclei, as in Figure 4. The attachment sites of osteoclasts are randomly ordered (arrowheads) when IRAG is deficient (top frames), whereas cells with scrambled siRNA retain normal attachment rings (arrows). ${ }^{1}$ Fields are $50 \mu \mathrm{m}^{2}$.
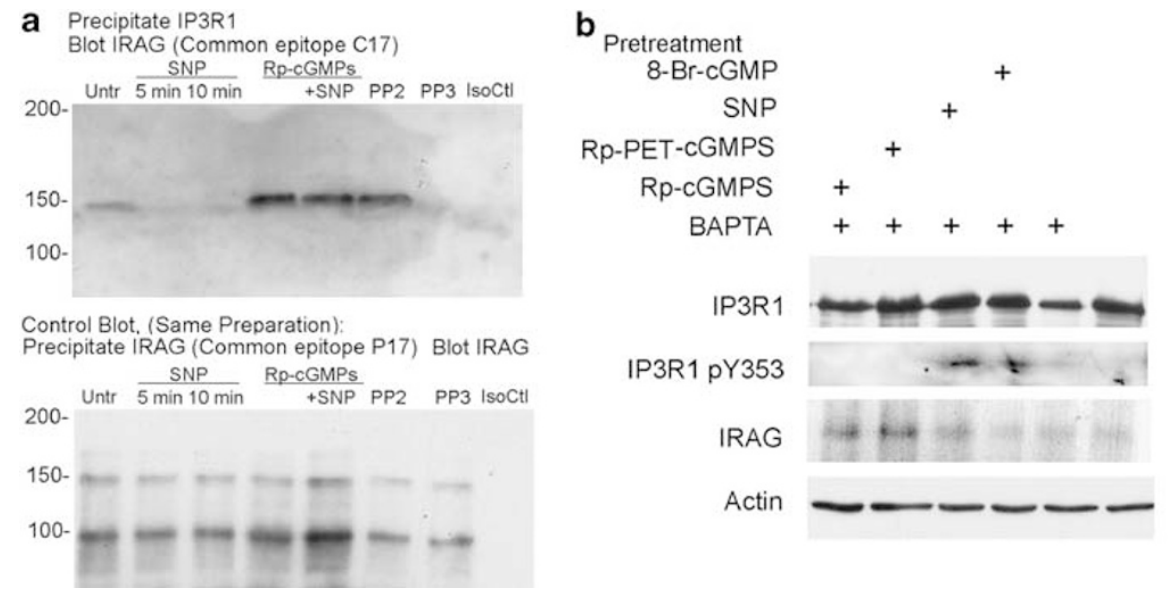

C Pretreatment

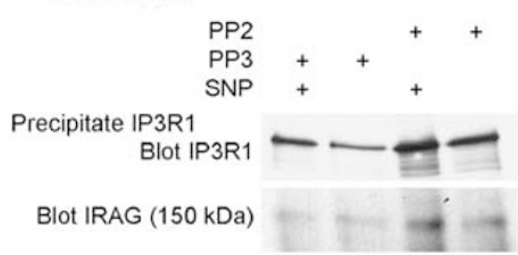

Western Blot of Supernatant

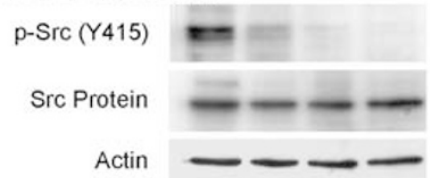

Figure 7 Association of IP3R with the $150 \mathrm{kDa}$ form of IRAG and effects of PKG or Src activity; IRAG not precipitated with IP3R1 binds cytoskeletal proteins. (a) Immune precipitation of IP3R1 followed by western analysis for IRAG. The $\sim 150 \mathrm{kDa}$ form of IRAG binds IP3R1, consistent with other reports. ${ }^{7}$ In keeping with immune localization (Figure 3), the IRAG-IP3R1 complex was inhibited by NO donors. The Src antagonist PP2, but not its inactive congener PP3, also stabilized the complex, even when sodium nitroprusside (SNP) was added. A control western blot from total lysate confirmed similar quantities of large and small forms of IRAG in this preparation (lower panel). (b) Studies precipitating IP3R1, then blotting using antibody to IP3R1 (top panel), stripping it, and reblotting for IRAG (third panel). Separate aliquots of the lysates were blotted directly for IP3R1 phosphotyrosine353 (second panel), followed by stripping and re-blotting for actin (bottom panel). Two cGMP inhibitors preserve IRAG-IP3R association (left two lanes) in preparations in which cell Ca ${ }^{2+}$ was held at a low level by BAPTA. In the NO donor sodium nitroprusside (SNP) and with the CGMP analog 8-Br-cGMP complexes were dissipated. With the NO donor or the CGMP analog, tyrosine phosphorylation of IP3R1 was observed (this is dependent on Src; see text and (c) below). (c) Immune precipitation of IP3R1 with western analysis for IP3R1, followed by stripping membranes and re-blotting for IRAG (top two panels). Separate aliquots of the supernatants were analyzed by western blot for phospho-Src tyrosine415; this blot was stripped and blotted for total Src and actin (bottom three panels). Strong Src phosphorylation occurred after sodium nitroprusside $(100 \mu \mathrm{m})$, but the Src antagonist PP2 eliminated this and, as in (a), IRAG-IP3R1 association was retained in NO donor cells when Src activity was eliminated. 
PKG1 $\beta$-binding site. ${ }^{5,8}$ Importantly, when the large form of IRAG is deficient, osteoclastic attachment is impaired and podosome distribution cannot be properly regulated. This defect is related, at least in part, to increased intracellular calcium and calcium-dependent proteinase activity. This defect is one potential cause for osteoclast attachment defects with normal attachment proteins, ${ }^{1}$ although other potential defects in pathways regulating attachment exist.

Our findings are consistent with phosphorylation of IRAG by PKG1 $\beta$ at serine residues, ${ }^{8}$ whereas tyrosine phosphorylation of IP3R1, activating the $\mathrm{Ca}^{2+}$ channel, depends on Src family kinases, probably mainly Src itself. Src is activated downstream of NO or cGMP signaling in osteoclasts, and $\mathrm{Src}$ knockdown attenuates $\mathrm{Ca}^{2+}$ signaling. ${ }^{2}$ The intermediate components of the Src activating pathway are unknown, and Src is not directly or solely activated by PKG1. Other reports have shown that inhibiting Src activity prevents osteoclast migration, ${ }^{21}$ in keeping with our work, but other mechanisms certainly regulate Src activity in osteoclasts. ${ }^{22}$ Thus, the effect of Src inhibitors on IP3R1 activation may reflect effects on diverse pathways as well as the NO-PKG1 $\beta$ mechanism.

An interesting novel observation was that $\mathrm{NO}$ or activating cGMP analogs caused disassociation of IRAG from IP3R1 (Figures 2 and 7). This effect, which was striking particularly when $\mathrm{Ca}^{2+}$ was held at low levels by chelation, may have been overlooked in other contexts because of rapid cycling and reconstitution of IRAG-IP3R1 complexes. Such rapid cycling is likely; PKG1 $\beta$ complexes in other contexts include phosphodiesterase-5. ${ }^{23}$ We also observed PKG-dependent nuclear localization of IRAG, in keeping with reports of its function in nuclear translocation of proteins, including of PKG. ${ }^{9}$ At least one nuclear transcription factor binds to the long form of IRAG ${ }^{24}$ suggesting that nuclear localization has complex functions. PKG1 itself regulates transcription of growth factors, including IL-1 and IL-6, in osteoclasts, ${ }^{25}$ but whether this requires IRAG is not known. Cytokines, including IL-1, conversely, modify NO signaling and $\mathrm{Ca}^{2+}$ response in the osteoclast, ${ }^{26}$ raising the possibility of transcriptional regulation of cellular $\mathrm{Ca}^{2+}$ signaling by a feedback mechanism.

As in other types of cells, ${ }^{27}$ we found that IRAG was required for cGMP-dependent $\mathrm{Ca}^{2+}$ release in osteoclasts. Some cells that express IRAG also express significant quantities of both PKG1 $\alpha$ and PKG1 $\beta,{ }^{28}$ only the latter interacting with IRAG. In contrast, we found that PKG1 $\alpha$ was a minor form of the enzyme in osteoclasts. The mechanism regulating specific processing of PKG1 $\beta$ is unknown. The $\mathrm{Ca}^{2+}$ fluxes regulated by PKG1 $\beta$ and IRAG may affect additional pathways, including other $\mathrm{Ca}^{2+}$ channels, ${ }^{19}$ although the extent to which this occurs is also unknown. However, it is clear that an essential element for modification of cell attachment by $\mathrm{Ca}^{2+}$ fluxes is $\mu$-calpain. ${ }^{2,29}$

It was observed over a decade ago that PKG modifies cell membrane-associated proteins in osteoclasts, ${ }^{30}$ although how cell membrane-associated proteins are selectively targeted was obscure. There are limited precedents for plasma membrane protein regulation by IRAG, although IRAG was identified by screening a membrane-related protein complex in tracheal epithelial cells. ${ }^{31}$ Although plasma membraneassociated IRAG was an unexpected discovery in the course of the present studies, this will be an important topic for further work. IRAG colocalized with membrane attachments after treatment with activating cGMP analogs (Figure 4), and, after precipitating IP3R1 complexes, IRAG precipitated from supernatant co-precipitated proteins including migfilin, VASP (Figure 4), and actin (not illustrated). These incidental observations are probably unrelated to the regulation of IP3R1, but they suggest that IRAG and its PKG1 $\beta$-binding domain $^{7}$ have multiple and disparate roles in osteoclast regulation, and probably regulate multiple biochemical pathways in other cells.

In summary, osteoclast IP3R1 is associated with the endosomal isoform of IRAG. IRAG is a target for phosphorylation by PKG1 $\beta$. In osteoclasts, PKG activity caused disassociation of the IP3R1-IRAG complex. Activation of $\mathrm{Ca}^{2+}$ release by IP3R1 after PKG activation depended on Src; the intermediate pathway for Src activation by NO and PKG is unknown. Additional observations included that PKG activation was associated with IRAG localization to cell attachments. Cells in which IRAG was reduced had randomly distributed podosomes, detached easily from substrate and had smaller average diameters, but remained viable. In these cells calpain activation still required PKG. We conclude that IRAG, and its modification by PKG1 $\beta$, have essential roles in osteoclast motility by regulating $\mathrm{Ca}^{2+}$ release.

\section{ACKNOWLEDGEMENTS}

We thank Professor Simon Watkins (University of Pittsburgh, Pittsburgh, PA, USA) for assistance with confocal microscopy and Professor Andrew Marks (Columbia University, NY, USA) for the antibody to IP3R

phosphotyrosine353. This study was supported by grants from the National Institutes of Health (USA) AR053976, AR055208, AR053566, and by the Department of Veteran's Affairs (USA).

\section{DISCLOSURE/CONFLICT OF INTEREST}

The authors declare no conflict of interest.

1. Blair HC, Yaroslavskiy BB, Robinson LJ, et al. Osteopetrosis with microlacunar resorption because of defective integrin organization. Lab Invest 2009;89:1007-1017.

2. Yaroslavskiy BB, Sharrow AC, Wells A, et al. Necessity of inositol $(1,4,5)-$ trisphosphate receptor 1 and $\mu$-calpain in NO-induced osteoclast motility. J Cell Sci 2007;120:2884-2894.

3. Morikawa K, Goto T, Tanimura A, et al. Distribution of inositol 1,4,5trisphosphate receptors in rat osteoclasts. Acta Histochem Cytochem 2008;41:7-13.

4. Kuroda $Y$, Hisatsune $C$, Nakamura $T$, et al. Osteoblasts induce $\mathrm{Ca}^{2+}$ oscillation-independent NFATc1 activation during osteoclastogenesis. Proc Natl Acad Sci USA 2008;105:8643-8648.

5. Schlossmann J, Ammendola A, Ashman K, et al. Regulation of intracellular calcium by a signalling complex of IRAG, IP3 receptor and cGMP kinase I $\beta$. Nature 2000;404:197-201.

6. Fritsch RM, Saur D, Kurjak M, et al. InsP3R-associated cGMP kinase substrate (IRAG) is essential for nitric oxide-induced inhibition of 
calcium signaling in human colonic smooth muscle. J Biol Chem 2004;279:12551-12559.

7. Shaughnessy JD, Largaespada DA, Tian E, et al. Mrvi1, a common MRV integration site in $\mathrm{BXH} 2$ myeloid leukemias, encodes a protein with homology to a lymphoid-restricted membrane protein Jaw1. Oncogene 1999;18:2069-2084.

8. Ammendola A, Geiselhšringer A, Hofmann $F$, et al. Molecular determinants of the interaction between the inositol 1,4,5trisphosphate receptor-associated CGMP kinase substrate (IRAG) and cGMP kinase I $\beta$. J Biol Chem 2001;276:24153-24159.

9. Casteel DE, Zhang T, Zhuang S, et al. cGMP-dependent protein kinase anchoring by IRAG regulates its nuclear translocation and transcriptional activity. Cell Signal 2008;20:1392-1399.

10. Antl $M$, von BrŸhl ML, Eiglsperger $C$, et al. IRAG mediates NO/cGMPdependent inhibition of platelet aggregation and thrombus formation. Blood 2007;109:552-559.

11. Murthy KS, Zhou H. Selective phosphorylation of the IP3R-I in vivo by cGMP-dependent protein kinase in smooth muscle. Am J Physio Gastrointest Liver Physiol 2003;284:G221-G230.

12. Cui J, Matkovich SJ, deSouza N, et al. Regulation of the type 1 inositol 1,4,5-trisphosphate receptor by phosphorylation at tyrosine353. J Biol Chem 2004;279:16311-16316.

13. Lee B, Vermassen E, Yoon SY, et al. Phosphorylation of IP3R1 and the regulation of $\left[\mathrm{Ca}^{2+}\right]_{i}$ responses at fertilization: a role for the MAP kinase pathway. Development 2006;133:4355-4365.

14. Marks AR. Intracellular calcium-release channels: regulators of cell life and death. Am J Physiol 1997:272:H597-H605.

15. Yaroslavskiy BB, Zhang $\mathrm{Y}$, Kalla SE, et al. NO-dependent osteoclast motility: reliance on CGMP-dependent protein kinase I and VASP. J Cell Sci 2005;118:5479-5487.

16. Yaroslavskiy BB, Li Y, Ferguson DJ, et al. Autocrine and paracrine nitric oxide regulate attachment of human osteoclasts. J Cell Biochem 2004:91:962-972.

17. Robinson LJ, Yaroslavskiy BB, Griswold RD, et al. Estrogen inhibits RANKL-stimulated osteoclastic differentiation of human monocytes through estrogen and RANKL-regulated interactions of estrogen receptor- $\alpha$ with BCAR1 and Traf6. Exper Cell Res 2009;315:1287-1301.

18. Steinmann C, Landsverk ML, Barral JM, et al. Requirement of inositol 1,4,5-trisphosphate receptors for tumor-mediated lymphocyte apoptosis. J Biol Chem 2008;283:13506-13509.
19. Robinson $\amalg$, Blair $\mathrm{HC}$, Barnett $\mathrm{JB}$, et al. Regulation of bone turnover by calcium-regulated calcium channels. Ann NY Acad Sci 2010;1192: 351-357.

20. Van Epps-Fung C, Williams JP, Cornwell TL, et al. Regulation of osteoclastic acid secretion by CGMP-dependent protein kinase. Biochem Biophys Res Commun 1994;204:565-571.

21. de Vries TJ, Mullender MG, van Duin MA, et al. The Src inhibitor AZD0530 reversibly inhibits the formation and activity of human osteoclasts. Mol Cancer Res 2009;7:476-488.

22. Nakajima A, Sanjay A, Chiusaroli $R$, et al. Loss of Cbl-b increases osteoclast bone-resorbing activity and induces osteopenia. J Bone Miner Res 2009;24:1162-1172.

23. Wilson LS, Elbatarny HS, Crawley SW, et al. Compartmentation and compartment-specific regulation of PDE5 by protein kinase $\mathrm{G}$ allows selective CGMP-mediated regulation of platelet functions. Proc Natl Acad Sci USA 2008;105:13650-13655.

24. Casteel DE, Boss GR, Pilz RB. Identification of the interface between cGMP-dependent protein kinase $\mathrm{I} \beta$ and its interaction partners TFIIand IRAG reveals a common interaction motif. J Biol Chem 2005;280:38211-38218.

25. Broderick KE, Zhang T, Rangaswami H, et al. Guanosine $3^{\prime}, 5^{\prime}$-cyclic monophosphate (cGMP)/cGMP-dependent protein kinase induce interleukin-6 transcription in osteoblasts. Mol Endocrinol 2007;21:1148-1162.

26. Bakker AD, Silva VC, Krishnan R, et al. Tumor necrosis factor- $\alpha$ and interleukin-1 $\beta$ modulate calcium and nitric oxide signaling in mechanically stimulated osteocytes. Arthritis Rheum 2009;60:3336-3345.

27. Frei $E$, Huster $M$, Smital $P$, et al. Calcium-dependent and Calciumindependent inhibition of contraction by $\mathrm{CGMP/CGKI}$ in intestinal smooth muscle. Am J Physiol Gastrointest Liver Physiol 2009; 297:G834-G839.

28. Geiselhšringer A, Gaisa M, Hofmann F, et al. Distribution of IRAG and cGKl-isoforms in murine tissues. FEBS Lett 2004;575:19-22.

29. Marzia $M$, Chiusaroli $R$, Neff $L$, et al. Calpain is required for normal osteoclast function and is down-regulated by calcitonin. J Biol Chem 2006;281:9745-9754.

30. Dong SS, Williams JP, Jordan SE, et al. Nitric oxide regulation of cGMP production in osteoclasts. J Cell Biochem 1999;73:478-487.

31. Koller A, Schlossmann J, Ashman $\mathrm{K}$, et al. Association of phospholamban with a CGMP kinase signaling complex. Biochem Biophys Res Commun 2003;300:155-160. 